\title{
Global risk of death due to delays in the care of pregnant women with obstetric complication in yucatan, Mexico
}

\begin{abstract}
Background: Delays in care of obstetric emergency exist. Studies involving specialists in charge of treating obstetric emergencies to get to know their perceptions on the care of maternal complications are scarce. There is evidence on the importance of taking into account the opinion of specialists in the face of complex problems, where the necessary information is difficult to come by with classical statistical methods.
\end{abstract}

Objective: To estimate the global risk of maternal death due to delays.

Methods: Elicitation technique with a panel of experts was used to elicit probabilities of delays regarding the care of the pregnant women with obstetric complications by means of Bayesian statistics. Four gyneco-obstetricians conformed the sample.

Results: The global risk of maternal death due to delays was $54 \%$. The final count of the global impact of mortality with the three-delay model in a real-life scenario was that $12.7 \%$ of the women with obstetric complication would die due to the presence of the three delays.

Conclusion: The global risk of death for delays in obstetric emergency care, obtained from the GOs', should be taken into account for decision-makers, in order to promote strategies to prevent delays and thus contribute to the reduction of maternal mortality.
Volume 9 Issue 2 - 2018

\author{
Elsa Rodr $\urcorner g u e z$ Angulo,' Guadalupe Andueza \\ Pech,' Rita Zapata Vÿzquez ${ }^{2}$ \\ 'Center of Regional Researchers, Dr. Hideyo Noguchi Social \\ Medicine and Public Health Laboratory, Mexico \\ ${ }^{2}$ Faculty of Medicine, University Autonomous of Yucatan, Mexico
}

Correspondence: Elsa Rodraguez Angulo, Center of Regional Researchers Dr. Hideyo Noguchia Calle 59 por Av, Itzaes Nm. 490, Col. Centro. CP 97000, M?rida, Yucatán, México, Email rangulo@correo.uday.mx

Received: February 12, 2018 | Published: March 21, 2018

\section{Background}

In 2015, for every 100,000 children born alive, 216 women died worldwide due to maternal causes. ${ }^{1}$ It is well known that pregnancy complications (preeclampsia/eclampsia, hemorrhage and sepsis), along with a chain of events cause delays in the care of obstetric emergencies and lead to death.,3 The greatest evidence on delays comes from descriptive studies carried out with pregnant women or their relatives. ${ }^{4}$ The studies that aim to specify the perceptions of specialists in charge of treating obstetric emergencies on the care of maternal complications are scarce. ${ }^{5,6}$ The three-delay model has been applied to the study of the non-medical causes of maternal deaths. It considers the analysis of the reasons that lead to a fatal outcome from a sociocultural perspective and from the angle of the access to obstetric emergency care. The first delay is attributed to the failure in recognizing the complications and looking for help; the second delay is due to the difficulty in finding a means of transportation to a hospital where the obstetric complication can be treated, and the third delay refers to the untimely treatment once the hospital has been reached. ${ }^{3}$ This chain of events ultimately leads to maternal death, and all the information gathered on the process comes from nonexpert people who do not work health personnel. There is evidence on the importance of considering the specialists' opinion in the face of complex problems which could not be managed with classical statistical methods. $^{7-9}$ The perception of the specialists in charge of treating the obstetric emergencies allows to have comprehensive information that complements the knowledge obtained at a community level. The elicitation technique with a panel of experts has been used to extract information that is difficult to get due to its complexity, but that can be useful in terms of health-related decisionmaking processes..$^{10-15}$ Since the issue of maternal death is related to multiple factors, ${ }^{16-18}$ the elicitation technique to extract a priori knowledge from gyneco-obstetricians (GOs) in charge of treating obstetric emergencies was applied in this study. The goal was to build a probabilistic model through a probability tree to estimate the global risk of maternal death due to delays. The perceptions on delays in the care of obstetric emergencies and their transformation in probabilities of death due to maternal complication is a measurable model of the global risk of maternal death in the state of Yucatan.

\section{Methods}

The participants in the study were specialized physicians who were experienced in taking care of pregnant women with obstetric complications. By means of a letter, GOs from the main health sector hospitals with the highest coverage of obstetric emergency care in Yucatan were invited to participate. The physicians had been working for at least five years; they had ongoing medical updating; they had basic knowledge of probability and statistics, and they were familiar with the procedures for treating pregnant women with obstetric complications, as well as with the referral and counter-referral mechanisms in place when the patients are sent from other localizations within the state. They were also willing to analyze the care procedures for pregnant women since the onset of the complication, the search for help and transportation, the arrival to the hospital and the final outcome (discharge or death). Furthermore, since the participating GOs were in charge of treating patients in public as well as in private institutions, both sectors in the state were duly represented. The sample consisted of four GOs (two women and two men) who were from one of the main referral hospitals in the state.

The aim of the study was explained before the elicitation process. The physicians were also told how their participation would be useful to determine the probability of delays in the case of pregnant women, which would in turn support the decision-making processes in order to improve care for patients. The participants were asked to sign a voluntary consent to participate in the panel. The starting point of 
reflection and conversation with the GOs was the analysis of the care procedures for pregnant women valid at the time of the study, taking as a reference point the Official Mexican Norm NOM-SSA2-1993. ${ }^{19}$ The care procedures described by the GOs were illustrated with graphics and figures with support of PowerPoint presentations.

The knowledge elicitation technique with a panel of experts was used to elicit probabilities of delays regarding the care of the pregnant women with obstetric complications by means of Bayesian statistics. ${ }^{20}$ The method was adapted to the participants' context and a facilitator, experienced in group management and knowledgeable about probability and statistics, guided the dialogue to elicit what the participants knew. The construction of the probabilistic model was carried out in four stages:

a. Creation of the probability elicitation instrument, which was adapted to the scenarios of the first, second and third delays. It was used to extract the a priori knowledge on the average of the delays resulting from the GOs' combination of probabilities.

b. Probability simulation exercise through which the participants were reminded what probabilities are and how they are estimated. It also allowed the facilitators to identify doubts and clear them up in a timely manner

c. Identification of probabilities of delay, first independently and then in a combined way.

d. Construction of the probability tree, with all the possible combinations of delays in order to determine the global risk of delay.

Through the elicitation process, the probabilities of delays were independently identified first. In other words, the probability of having experienced the first, second or third delay was specified initially. Next, the probability of death of each of the possible combinations was determined. To elicit the probability of death due to delays, the question was: What is the probability of death of pregnant women with some kind of complication? At the end of the process, the median and its ranges were determined for the first, second and third delays. Using Excel, tables including the question asked to the experts, the upper range of probability, the lower range of probability, the highest probability (median), and the specific probabilities of each delay were built. The probabilities of death that were elicited were represented on a probability tree.

The procedure used to estimate the probabilities of dying, using TreeAge Pro $12,{ }^{21}$ consisted in a rollback calculation, where the probabilities are added and multiplied by all the possible combinations of delays. The probability tree was presented to the specialists, who analyzed whether those probabilities represented what they had expressed. Duration of each session was maximum four hours. When time ran out before the session was concluded, another session was scheduled with each specialist. An elicitation process was carried with each specialist. When these sessions were done, the group was gathered to allow the discussion of experiences and to get feedback on the information given at the individual sessions. The goal was to arrive at a consensus regarding the distribution of the probabilities elicited.

Finally, the global probability of death due to delays in patients with obstetric complications was obtained. The distribution of the impact of that probability in a real-life scenario of death and survival among pregnant women that have been exposed to all the possible combinations regarding delays in the care of obstetric complications was also determined.

\section{Results}

The age ranged of the GOs was from 36-70 years, two of them had more than thirty years on service. The elicited probabilities of experimenting any delay due to obstetric complication were 0.391 $(39.1 \%)$ for the first delay, $0.602(60.2 \%)$ for the second delay, and $0.599(59.9 \%)$ for the third delay. The highest elicited probabilities of death due to delays were obtained when the three delays were combined $(0.90[90 \%])$ and when the first and third delays were combined $(0.75[75 \%])$, but not the second. The probability of global death for pregnant women when probabilities of death and survival were combined was 0.54 (54\%) (Figure 1).

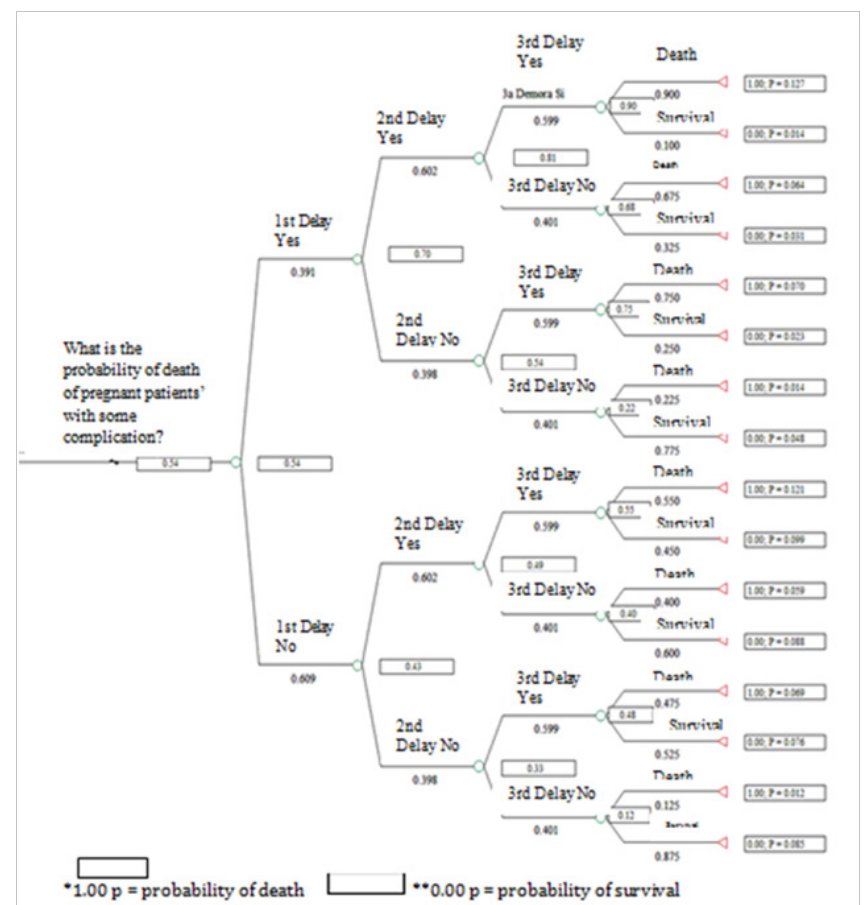

Figure I Probability tree of the three-delay model regarding the care of pregnant women with obstetric complications.

Table 1 summarizes the probabilities of dying, extracted from Figure 1 (rectangles to the right of the circular nodes, located to the right of the tree) corresponding to the three-delay model. The highest probabilities of death (marked in bold) occur when the pregnant woman is exposed to the three delays $(0.90)$, to the first and third delays $(0.75)$, to the first and second delays $(0.68)$, and to the second and third delays $(0.55)$. In other words, the highest probabilities of death occur in the presence of the three delays or with the combination of two of them. The probability of death is higher in the case of the third delay alone (48\%), compared to suffering from the second delay alone $(40 \%)$ or only the first delay $(22 \%)$. It is noteworthy that the first delay is present in the three highest probabilities of mortality.

\section{Final impact of delays}

\section{Probabilities of death:}

The final count of the global impact of mortality (54\%) with the three-delay model in a real-life scenario was that $12.7 \%$ (first rectangle to the right) of the women with obstetric complication would die due to the presence of the three delays; whereas only $1.4 \%$ of the women suffering from the three delays would survive (Table 2).

About $6.4 \%$ (third rectangle to the right) of deaths corresponds to pregnant women that suffered from the first and second delays 
whereas $3.1 \%$ (fourth rectangle to the right) of the pregnant women with the same background survived. The final impact of the third delay when the previous two have been present is immense: $12.7 \%$ of dead women compared to $6.4 \%$ (when the third delay is absent). In other words, there are twice as many deaths in the presence of the third delay.

Table I Probabilities of death for pregnant women with obstetric complication with the three-delay model

\begin{tabular}{llll}
\hline Probability & Delay I & Delay 2 & Delay 3 \\
\hline 0.9 & $\sqrt{ }$ & $\sqrt{ }$ & $\sqrt{ }$ \\
0.75 & $\sqrt{ }$ & - & $\sqrt{ }$ \\
0.68 & $\sqrt{ }$ & $\sqrt{ }$ & - \\
0.55 & - & $\sqrt{ }$ & $\sqrt{ }$ \\
0.48 & - & - & $\sqrt{ }$ \\
0.4 & - & $\sqrt{ }$ & - \\
0.22 & $\sqrt{ }$ & - & - \\
0.12 & - & - & -
\end{tabular}

Table 2 Final scenario of death and survival percentages with the three-delay model

\begin{tabular}{lllll}
\hline \% Death & \% Survival & I st D & 2nd D & 3rd D \\
\hline 12.7 & I.4 & $\sqrt{ }$ & $\sqrt{ }$ & $\sqrt{ }$ \\
6.4 & 3.1 & $\sqrt{ }$ & $\sqrt{ }$ & - \\
7 & 2.3 & $\sqrt{ }$ & - & $\sqrt{ }$ \\
1.4 & 4.8 & $\sqrt{ }$ & - & - \\
12.1 & 9.9 & - & $\sqrt{ }$ & $\sqrt{ }$ \\
5.9 & 8.8 & - & $\sqrt{ }$ & - \\
6.9 & 7.6 & - & - & $\sqrt{ }$ \\
I.2 & 8.5 & - & - & - \\
Total: $54 \%$ & Total: $46 \%$ & & &
\end{tabular}

i. $7 \%$ of the dead women would have suffered from the third and first delays, but not from the second; and only $2.3 \%$ of the survivors would have the same background. On the other hand, $1.4 \%$ of the dead women would have suffered from the first delay, and $4.8 \%$ of the survivors would have suffered only from the first one. When comparing the final probability of death among those women who suffered from the first and third delay, but not the second $(7 \%)$, with those that only suffered from the first delay $(1.4 \%)$, mortality was five times higher among the former than among the latter.

ii. $12.1 \%$ of the dead women had suffered from the third and second delays, and $9.9 \%$ would survive with the same background. However, $5.9 \%$ of the dead women had suffered only from the second delay; whereas $8.8 \%$ of the survivors would have likewise suffered only from the second delay. Mortality increased 2.05 times due to the fact of having suffered from two delays (third and second), compared to the cases that only suffered from the second one.

iii. Death in $6.9 \%$ corresponded to those women who suffered only from the third delay; whereas $7.6 \%$ corresponded to the survivors in the same condition. However, only $1.2 \%$ of the women would be dead without any delay (in other words, a thousand pregnant women with some kind of complication would be necessary in order for 12 deaths to occur in the absence of all the delays), and $8.5 \%$ of the women who did not suffer from any delay would survive. iv. Ultimately, when comparing mortality in the final scenario, the probability of death in the presence of the three delays (12.7\%) (First rectangle to the right of the terminal nodes), decreased 10.58 times (to $1.2 \%$ ) when the delays were absent (last rectangle to the right of the terminal nodes).

\section{Probabilities of survival}

a. In terms of survival, when comparing the branch with the three delays (1.4) (second upper branch of the tree) with the branch without any delay (8.5) (last lower branch of the tree), survival turned out to be 6.07 times higher in the absence of delays, than in the presence of the three delays together (Table 2).

b. In this scenario, it can be observed that the probability of survival is $3.1 \%$ in the presence of the first and second delays, but not the third. In the presence of the third delay, but not the first and the second, the probability of survival is $7.6 \%$, that is to say, 2.45 times more compared with the women who did not suffer from the third delay, but suffered from the first and second.

c. If we compare the $2.3 \%$ survival probability in the presence of the first and third delays but not the second, with the $8.8 \%$ survival probability in the sole presence of the second delay, there is a 3.82 increase in the probability of survival when only the second delay is present.

d. Survival is higher $(9.9 \%)$ when the first delay is absent, even though the second and third are present, compared to survival when only suffering from the first delay $(4.8 \%)$. Then, the probability of survival increases 2.06 times in the absence of the first delay.

e. When the first delay was present, but not the second nor the third, women survived less $(4.8 \%)$ than those who suffered from the second delay, but not from the first and the third $(8.8 \%)$, and less than those who suffered from the third delay, but not from the second and first $(7.6 \%)$. The survival of women who suffered from the first and second delays but not the third (3.1\%) was lower than the survival of those who suffered from the first delay but not from the second or the third $(4.8 \%)$. This means, that the probabilities as the delays occur have an impact on the final scenario, as was observed in the case of suffering from the second delay, which was more probable $(60.2 \%)$ than not suffering from it $(39.8 \%)$. This affected the probability of survival: $32.5 \%$ vs $77.5 \%$, respectively. Survival was also higher among those women who suffered from the second and third delays but not from the first $(9.9 \%)$ and among those who suffered from the third delay but not from the second or the third (7.6\%).

f. When comparing survival in this scenario, there would be more survivors in the presence of the second delay alone (8.8\%), than with the third $(7.6 \%)$ or the first $(4.8 \%)$. This has to do with the frequency with which the second delay occurs, although mortality related to it is two times that of the first delay.

\section{Discussion}

The elicitation technique with a panel of experts allows defining the global risk of death due to delays in the treatment of pregnant women with obstetric complications. Although every day the number of studies using this technique increases, ${ }^{13,22,23}$ we did not find any other study where the technique was applied to elicit the probabilities of delay regarding maternal mortality. Therefore, our results set a precedent for the use of the methodology in future studies related 
to this complex issue, as well as in other research topics for which information is not available due to lack of studies, to its high costs, or because information is difficult to reproduce owing to health or environmental risks.

The construction of the probability tree regarding death due to delays in the care of pregnant women with obstetrical complications allows identifying the magnitude of the harm to which women are exposed. It was demonstrated that the more delays occur in the process, the greater the risk of death is. The model combined all the possible situations that pregnant women with obstetric complications can undergo, whether they are exposed to delays (single or combined) or not. It is noteworthy that the third delay, alone or combined, appeared in three of the four highest probabilities of death. The probability of death of pregnant women with obstetric complications increased as the delay is prolonged. The presence of the third delay in three out of the four highest probabilities implies acknowledging the responsibility of health system about implementing measures that improve the quality of care for obstetric complications in hospitals. This also implies the revision of the referral and counter-referral system of women, since many of them come from the state's inner municipalities, where there are not enough resources to treat the emergency. This situation prevails as one of the strong barriers to timely care of obstetric complications..$^{24}$

The presence of the first delay along with the third resulted in a high probability of death (75\%). In the year 2012 in Yucatan, the combination of these two delays resulted in a lower percentage (67\%) of deaths due to maternal cause in six southern state municipalities. ${ }^{25} \mathrm{In}$ that same study, gender-related conditions were present, such as the lack of decision-making power of the pregnant women regarding who should take care of their deliveries. In $89 \%$ of the cases, their mothers or mothers-in-law made the decisions. In another study carried out in Yucatan, the existence of communities with deeply ingrained traditions was shown: Women have been socialized to do only what their husband's say. ${ }^{26}$ Therefore, it is necessary that the causes underlying the first delay should be considered to implement strategic prevention measures. The focus would not only be to raise awareness of the alarm signs of the main maternal complications, but also to acknowledge the importance of reproductive rights and gender equality in order to reduce the delays once the complication has occurred.

It is necessary to continue engaging specialists to participate in futures studies to address the issue of maternal mortality in Yucatan. Their experience translated into probabilities, reflect the magnitude of the harm, thus reinforcing the results of the qualitative studies. ${ }^{24}$ It will also be necessary to revise the public policies implemented to prevent maternal mortality in Yucatan and to propose strategies to improve the quality of obstetric emergency care. The goal is to reduce delays and increase the survival probability of patients.

\section{Conclusion}

The global risk of death for delays in obstetric emergency care, obtained from the GOs', should be taken into account by decisionmakers, in order to promote strategies to prevent them and thus contribute to the reduction of maternal mortality in Yucatan.

\section{Acknowledgements}

The authors thank the gyneco-obstetricians that participated in this study for having offered their time and shared their experiences to obtain probabilities.

\section{Conflicts of interest}

The authors declare that they do not have any conflicts of interest.

\section{Ethical approval}

Ethical approval for this study was granted by the Bioethics and Research Committee at the "Dr. Hideyo Noguchi" Regional Research Center in the Autonomous University of Yucatan.

\section{Consent}

After brief explanation of the purpose of the study, written consent was obtained from the participants. All of them voluntarily signed an informed consent form, and they had the right to withdraw from the study at any time.

\section{References}

1. Alkema L, Chou D, Hogan D, et al. Global, regional, and national levels and trends in maternal mortality between 1990 and 2015, with scenario-based projections to 2030: a systematic analysis by the UN Maternal Mortality Estimation Inter-Agency Group. Lancet. 2016;387(10017):462-74

2. GBD 2013 Mortality and Causes of Death Collaborators. Global, regional, and national age-sex specific all-cause and cause-specific mortality for 240 causes of death, 1990-2013: a systematic analysis for the Global Burden of Disease Study 2013. Lancet. 2015;385(9963):11771.

3. Thaddeus S, Maine D. Too far to walk: maternal mortality in context. Soc Sci Med. 1994;38(8):1091-110.

4. Rodríguez-Angulo E, Palma-Solís M, Zapata-Vázquez R. Causes of delay in the care of patients with obstetric complications? what is necessary to attend? Ginecol Obstet Mex. 2014;82:647-658.

5. Demaria L, Campero L, Vidler M, et al. Non-physician providers of obstetric care in Mexico: Perspectives of physicians, obstetric nurses and professional midwives. Hum Resource Health. 2012;10:6.

6. Walker D, DeMaria L, Suarez L, et al. Skilled birth attendants in Mexico: how does care during normal birth by general physicians, obstetric nurses, and professional midwives compare with World Health Organization evidence-based practice guidelines? J Midwifery Womens Health. 2012;57(1):18-27.

7. Molinero LM. Spain: The Bayesian Method in Medical Research.

8. Meyer M, Booker J. Eliciting and analysing expert judgment. A practical guide. Society for industrial and applied mathematics. American statistical association. Londres: Academic Press Limited, 1991; 491 p.

9. Hoffman RR. Human factors contributions to knowledge elicitation. Hum Factors. 2008;50(3):481-8.

10. Leal J, Wordsworth S, Legood R, et al. Eliciting expert opinion for economic models: an applied example. Value Health. 2007;10(3):195-203.

11. Umoquit MJ, Dobrow MJ, Lemieux-Charles L, et al. The efficiency and effectiveness of utilizing diagrams in interviews: an assessment of participatory diagramming and graphic elicitation. BMC Med Res Methodol. 2008;8:53.

12. Stevenson MD, Oakley JE, Lloyd Jones M, et al. The cost-effectiveness of an RCT to establish whether 5 or 10 years of bisphosphonate treatment is the better duration for women with a prior fracture. Med Decis Making. 2009;29(6):678-89.

13. Saunders M, Magnanti BL, Correia Carreira S, et al. Chlorpyrifos and neurodevelopmental effects: a literature review and expert elicitation on research and policy. Environ Health. 2012;11 Suppl 1:S5. 
14. Brett Hauber A, Fairchild AO, Reed Johnson F. Quantifying benefit-risk preferences for medical interventions: an overview of a growing empirical literature. Appl Health Econ Health Policy. 2013;11(4):319-29.

15. Bowler RM, Beseler CL, Gocheva VV, et al. Environmental exposure to manganese in air: Associations with tremor and motor function. Sci Total Environ. 2016;541:646-54.

16. Health and nutrition. UNICEF report. UNICEF Strategy in Latin America and the Caribbean to contribute to the reduction of maternal, neonatal and child morbidity and mortality. 2011-2015.

17. Social determinants. Ministry of Health. Government of Chile.

18. Barkat A, Rahman M, Bose ML, et al. Modelling the first two delays of the "three delays model" for emergency obstetric care in Bangladesh: a choice model approach. J Health Popul Dev Ctries. 1997;1(1):57-67.

19. Current NOMs. Important information. Official Mexican Standard NOM-SSA2-1993.

20. Gartawaite PH, Kadane JB, O Hagan A. Statistical methods for eliciting probability distributions. 2005.
21. TreeAge Pro Software, inc, 2012.

22. Davies A, Vardeva K, Loze JY, et al. Cost-effectiveness of atypical antipsychotics for the management of schizophrenia in the UK . Curr Med Res Opin. 2008;24(11):3275-85.

23. Karnon J, Czoski-Murray C, Smith KJ, et al. A hybrid cohort individual sampling natural history model of age-related macular degeneration: assessing the cost-effectiveness of screening using probabilistic calibration. Med Decis Making. 2009;29(3):304-16.

24. Rodríguez EM, Oliva Y, Andueza MG, et al. Physicians' perceptions of the processes, barriers and strategies in the care of obstetric complications. J Women's Health Gyn. 2017;4:203-212.

25. Rodríguez-Angulo E, Montero-Carvantes L, Andueza-Pech G, et al. Medical-social characteristics of maternal deaths in a mayan community of yucatan, Mexico. Ginecol Obstet Mex. 2007;75(2):79-85.

26. Rodríguez E, Aguilar P, Montero L, Hoil J, Andueza G. Delays in the care of maternal complications associated with deaths in municipalities in southern Yucatán, Mexico. Rev Biomed. 2012;23:23-32. 